\title{
Homogenous multi-agent optimization for process systems engineering problems with a case study of computer aided molecular design
}

\author{
Berhane H. Gebreslassie, Urmila M. Diwekar*
}

\begin{abstract}
In this paper, we propose a novel homogenous multi-agent optimization (HMAO) framework for optimal design of large scale process system engineering problems. The platform is validated using a benchmark problems and a computer-aided molecular design (CAMD) problem. The molecular design problem is a solvent selection problem and it is formulated as a mixed integer nonlinear programming (MINLP) in which solute distribution coefficient of a candidate solvent is maximized subject to structural feasibility, thermodynamic property and process constraints. The model simultaneously determines the optimal decisions that include the size and the functional groups of the candidate solvents. In developing the HMAO framework, multiple efficient ant colony optimization (EACO) algorithms are considered as distinct algorithmic agents. We illustrate this approach through a real world case study of the optimal design of solvent for extraction of acetic acid from waste process stream using liquid-liquid extraction. The UNIFAC model based on the infinite dilution activity coefficient is used to estimate the mixture properties. The results show that quality of the objective function and the computational efficiencies are improved by a factor ranged from 1.475 to 4.137 . The new solvents proposed in this work are with much better targeted thermodynamic properties compared to the solvents proposed so far in previous studies.

Keywords: Multi-agent optimization, computer aided molecular design, hammersley sequence sampling, oracle penalty function, UNIFAC
\end{abstract}




\section{Introduction}

A wide range of optimization problems that include a large number of continuous and/or discrete decision variables, fall into the category of linear programming (LP), nonlinear programming (NLP), integer programming (IP), mixed integer linear programming (MILP), mixed integer nonlinear programming (MINLP) and disjunctive programming problems. Gradient based methods such as Branch and Bound (BB), Generalized Bender's Decomposition (GBD), and Outer Approximation (OA), are generally used for solving IP, MILP, and MINLP problems. However, these methods have limitations whenever the optimization problems fail to satisfy convexity property, the problems have large combinatorial explosion, or when the search domain is discontinuous (Diwekar and $\mathrm{Xu}, 2005$ ). Metaheuristic optimization strategies such as simulated annealing (SA) (Kirkpatrick et al. 1983), genetic algorithm (GA) (Holland, 1975) and ant colony optimization (ACO) (Dorigo 1992) can provide a viable alternative to the gradient based programming techniques. These individual optimization algorithms have a unique problem solving property. These standalone algorithmic procedures can solve certain types of problems effectively and may fail to progress for other types of problems Siirola (2003). Meta-heuristics do not, in general, guaranty optimality and the performance often depends on the particular problem characteristics and parameter setting. Therefore, a major issue in meta-heuristic design and calibration is to build them for maximum performance and make them robust. In this context, designing the standalone meta-heuristics is offering a consistently high level of performance over a wide variety of problem settings and characteristics Crainic and Toulouse (2003).

Multi-agent optimization (MAOP) framework provides a way of combining standalone deterministic and stochastic algorithmic procedures in one platform and exploits the strength possessed by each algorithm. Therefore, unlike a standalone method (only one strategy), the optimization problem in MAOP framework is approached with different solution methods of different background and with different parameter settings. In this context, contrary to the standalone optimization algorithm, the MAOP strategy avoids the problem of getting stuck in local optima. The optimizing agents use different algorithms to solve the same problem. These agents could be running on different machines or within one multicore system machine in parallel. Because of the collaboration among each agent through the global sharing memory, every agent will be initialized with improved findings of the other agents at each iteration and 
hence the individual agents will perform smaller number of iterations than typically needed to reach the optimal solution that lead to shorter elapsed time.

Because of the diversified nature of the algorithmic procedures involved in developing the MAOP framework, it has the capability of solving problems with different complexity and background. In this work, we propose a multi-agent optimization framework for solving large scale process system engineering problems. The proposed MAOP framework uses a variety of multiple algorithmic agents which include multiple efficient ant colony optimization (EACO) algorithms that differ in the initialization of the solution archive and the algorithmic parameters setting. Each agent encapsulates a particular range of parameters. We investigate the effect of cooperation among agents of the multi-agent system working in parallel, in series and standalone combined into a framework designed to solve large scale discrete, continuous and combinatorial optimization problems. Computational experiments are carried out using benchmark problems and a real world case study.

The rest of the paper is organized as follows: The following section describes the proposed MAOP framework and discusses the effect of diversity, coordination and parallelization of agents in the MAOP framework. In section 3, the efficient ant colony algorithm is introduced. In section 4, benchmark problems and results are introduced and discussed. The computer aided molecular design problem formulation is discussed in section 5. In same section, the results of the solvent selection case study is presented and discussed. Finally, the concluding remarks are presented in section 6 .

\section{Literature review and description of multi-agent optimization}

Multi-agent optimization is a nature-inspired optimization method, which supports cooperative search by group of algorithmic agents connected through an environment with certain predefined information sharing protocol. An agent in the MAOP framework is formed by combining an algorithmic procedure, a communication protocol between the algorithmic procedure and the global information sharing environment, the algorithmic procedure specific initialization and output retrieving methods. In this context, an agent can be defined as a distinct, autonomous software entity that is capable of observing and altering its environment. An agent evaluates a given task that contributes directly or indirectly to the advancement of it's surrounding Siirola et al (2003). The agents are combined into a cohesive system where the 
individual agents interact through the global information sharing environment. The MAOP framework exhibits both the aggregate properties of the individual agents, and superior properties resulting from the interactions among the individual agents. In this nature inspired MAOP platform, the overall behavior is not governed by a strong, centralized control rather by the individual algorithms that make up the framework operate autonomously, and a better overall system behavior emerges from the interactions among the individual agents through the global sharing environment Siirola et al (2003). Hence, the MAOP framework has the capability to solve problems from different background with better computational efficiency.

Multi-agent systems have been used in many modeling and simulation studies. However, the potential to use the multi-agent approach as global optimization strategy has been limited to small benchmark problems. In process systems engineering, agent-based systems are proposed for conceptual design (Han et al. 1995), supply chain management (Julka et al. 2002a, Julka et al. 2002b, Mele et al. 2007), and controller design (Tatara et al. 2005, Tetiker et al. 2008). However, related to multi-agent optimization for process systems engineering problems, very few articles have appeared and most of them are restricted to small scale problems (Siirola et al. 2003, Siirola et al. 2004). In this work, the multi-agent optimization framework will be developed to solve large-scale and real-world optimization problems. The flow of data and control structure will be similar to Siirola et al. (2003). However, the agents will be designed differently for solving large scale optimization problems. Siirola et al. (2003) proposed a modular framework for implementing agent-based systems for nonconvex engineering design optimization. The multi-agent framework has successfully integrated several agents to solve a single objective optimization and the result analysis indicate that the proposed framework to work well as a candidate framework for solving nonlinear optimization problems. They have concluded that by effectively combining the deterministic, stochastic, and heuristic methods into a system, it can be achieved a system that out-performs any of the methods working in isolation, without the need to develop an explicit policy for information sharing. The framework proposed by Siirola et al. (2003) is based on A-Teams concept of Talukdar et al. (1998). A-teams architecture is a set of autonomous agents and memories that are connected through a cyclic network to solve an optimization problem by modifying one another's trial-solutions. Each agent uses its algorithmic procedure to improve the solutions selected from its input-memory. In each iteration, every agent performs the same three steps: select a solution from a population; modify 
the selected solution though the application of the algorithmic procedure; then send back the modified solution into the solution population. To provide cooperation, input and output memories of agents are connected through communication channels so that an agent may select the output of another agent as its input. The system terminates when a persistent solution appears. Recently, Aydemir et al. (2013) proposed a multi-agent cooperation for solving global optimization problems through the introduction of a new multi-agent environment, MANGO. MANGO is a Java-based multi-agent global optimization framework that provides an environment to deploy software agents that cooperatively solve global optimization problems. Moreover, it provides fundamental facilities for users to implement their own agents to run in a MANGO environment. The central principle of MANGO is the effective use of cooperation among agents by providing a communication mechanism in which agents can exchange messages to share their information. The authors show how the multi-agent structure is more effective than having a single nonlinear optimization algorithm with randomly selected initial points. However, MANGO is designed only to solve continuous optimization problems.

Process system engineering problems are large scale problems and they include discontinuous, nonlinear and nonconvex problems with multiple local optima solutions. The MAOP framework is well-suited to solving process system engineering problems. The goal of this work is to develop a MAOP framework and solve a process system engineering problem into its global optimal solution which is a combination of local objective functions and respective model constraints accessed only by the local algorithmic procedures (agents).

The major steps of the MAOP framework includes representation of the optimization problem, the global sharing memory environment, pool of algorithmic agents, scheduler that allocate resource and execution of the algorithmic agents to solve the task assigned to them, processor and retrieving the final solution. The basic flow diagram for this framework is given in figure 1. The proposed methodology enables to improve the quality of solutions and the computational efficiency in comparison with multi-agent framework solved in series and standalone agents.

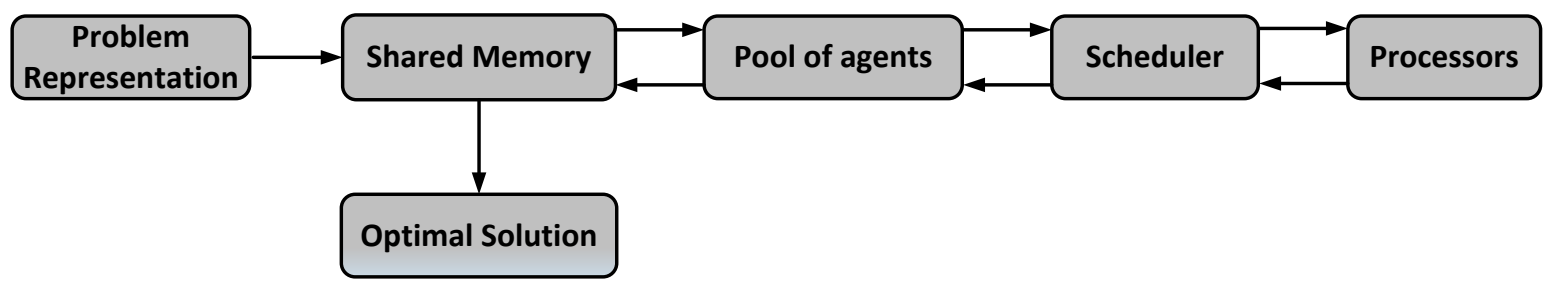


Figure 1: The generic form of Multi-agent optimization (MAOP) framework

The major emphasis of MAOP framework are enhancing the accommodation of different classes of optimization problems and increasing the computational efficiency. Therefore, the MAOP focuses on the diversity of agents involved in the framework, the coordination between agents and the global sharing memory for a better cooperation and the parallelization of agents.

\subsection{Diversity}

Depending on the type of agents included in the agent pool of the MAOP framework, MAOP is classified as homogenous and heterogeneous MAOP framework. The homogeneous MAOP (HMAO) only considers agents with similar feature. In this case, agents differ only in terms of the algorithmic parameters and the initialization. Because of the similarity in background of the agents, this search algorithm platform may be successful for certain types of problems and fail to progress for other types of problems. To circumvent this difficulty instead of using algorithmic procedures of similar background, the heterogeneous MAOP (HTMAO) framework uses diverse search algorithms. The HTMAO scheme allows to combine several algorithms with different features such as combining the gradient based algorithms and the different class of metaheuristic algorithms in one platform. Thus, the strength of one agent can be exploited to overcome the weakness of the other agents for the common goal of getting the global optimal solution. The two figures (figures $2 \mathrm{a}$ and $2 \mathrm{~b}$ ) illustrate the two MAOP schemes. The two algorithm schemes are identical in terms of the coordination and parallelism. In this work, we are focused on the HMAO platform and the efficient ant colony algorithm (EACO) Gebreslassie and Diwekar $(2015,2016)$ is used as an algorithmic procedure. The algorithmic parameters of each agent considered can be viewed in the first paragraph of the result and discussion. 


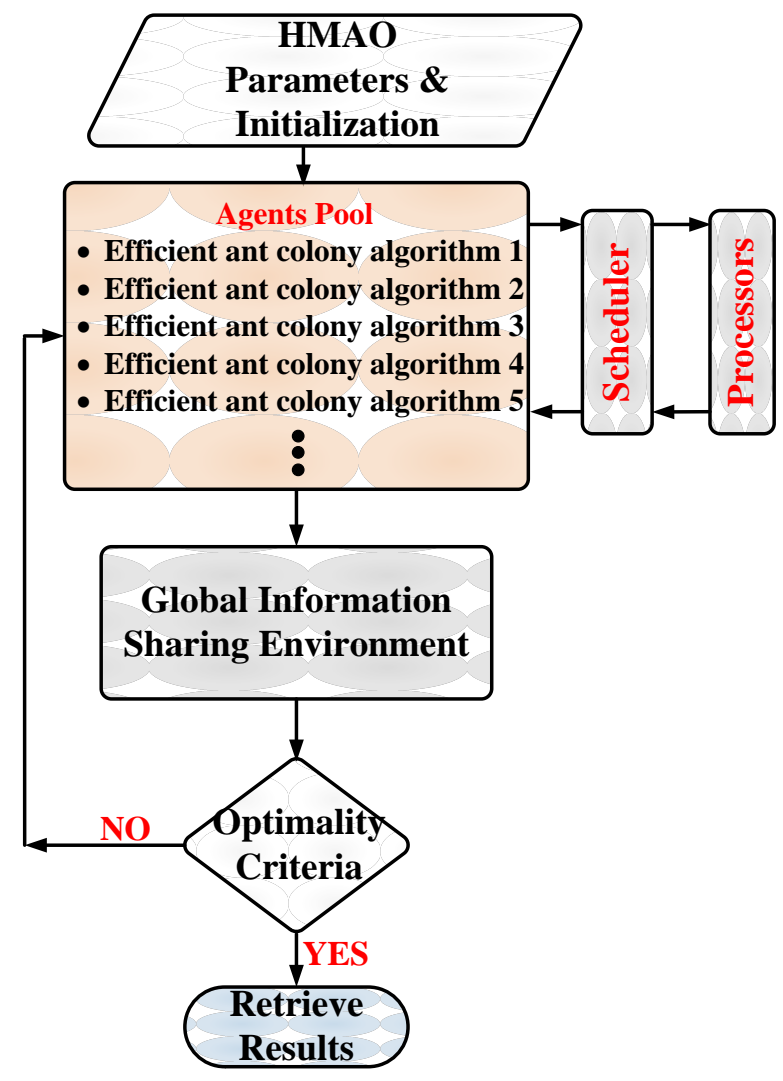

(a)

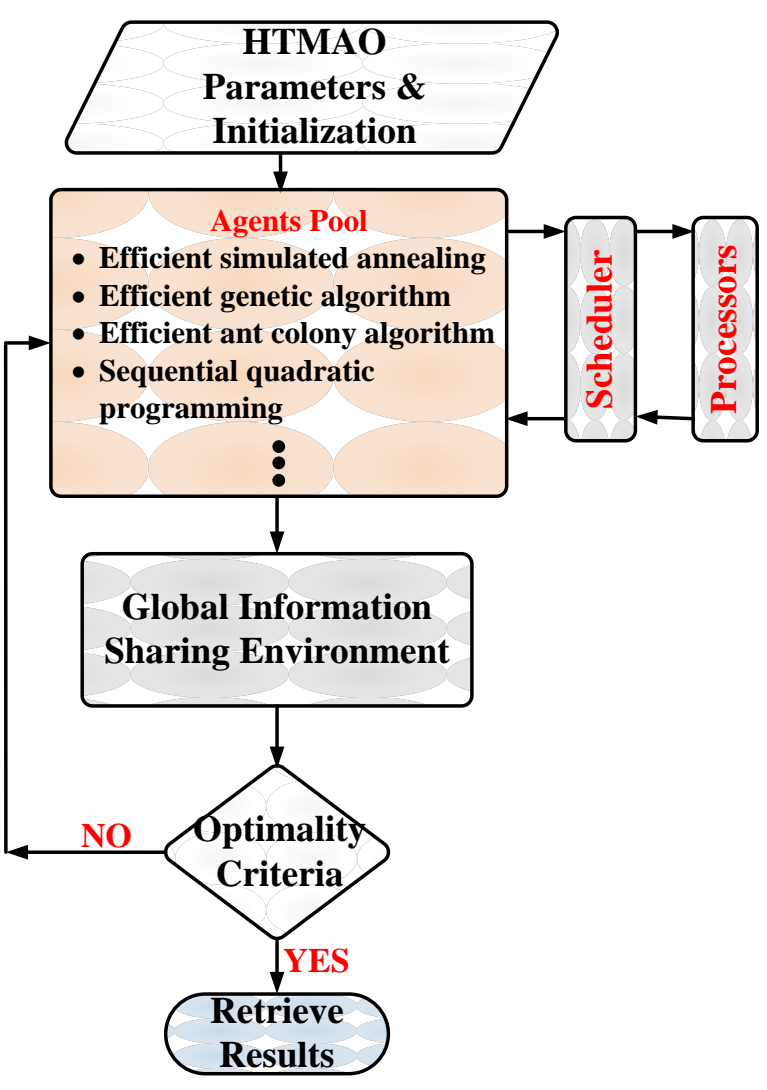

(b)

Figure 2: Homogenous (a) and Heterogeneous (b) multi-agent optimization frame works.

\subsection{Coordination}

There are two major coordination mechanisms in the multi-agent optimization literature which are the task and result sharing mechanisms. In the task sharing, a problem is decomposed into smaller sub-problems and each sub-problem is assigned to an agent by a central coordinating agent. In this case, it is a modular coordination in which an agent operation is dependent on the results of other agents. Depending on the complexity of the problem, both the task division and the assignment of tasks to agents with different capabilities is not trivial. If the tasks are independent, the problem can be concurrently solved by several agents. Then, the agents can share their results and refine the optimal solution of the problem. The result sharing coordination mechanism is considered better over task sharing since it allows agents to cross-check results within the local memory and the global memory Aydemir et. al. (2003). The coordination system implemented in this work resembles to the MANGO coordination mechanism which is based on 
message passing related to agent results and global solutions. To preserve the autonomy of agents, each agent establishes a specific communication protocol with the global sharing environment based on how fits best to the agent specific needs. In this context and as shown in figure 3, an agent in the MAOP framework is formed by combining an algorithmic procedure, the agent specific communication protocol between the algorithmic procedure and the global information sharing environment, the algorithmic procedure specific initialization and output retrieving methods.

The type of message exchanged between an agent and the global sharing memory differ from agent to agent. As the agents are autonomous and having different background, they are free to choose what type of message to exchange according to their need. Agents use the information gained from the global sharing memory to find better solutions and post new information to the global sharing memory to enhance the search of the global optimal solution.

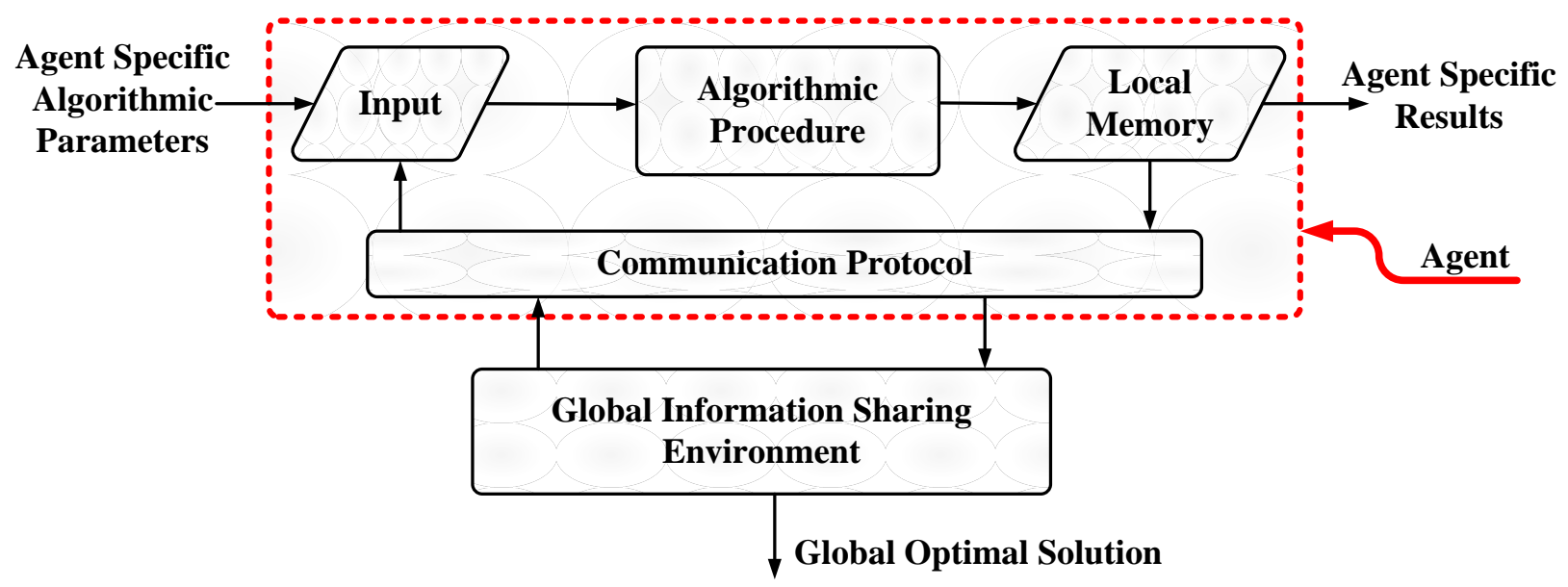

Figure 3: An agent in MAOP framework

The algorithmic procedure of an agent solve a problem using specific inputs such as the initialization, algorithmic parameters from the input memory and places the results in the local output memory. The communication protocol of an agent decides which objects will be retrieved from the local output memory and posted into the global sharing memory. There could be some results from the algorithmic procedure which are only accessed by the local sharing memory. Therefore, the communication protocol of an agent is consists of selectors that selects data from the global sharing memory according to the need of the agent and post to the input memory for the use in the agent algorithm operation, and it also selects the objects that will be posted in the global sharing memory from the local output memory. Moreover, it is used as an agent identifier 
that will link with the scheduler for the execution and resource allocation of the agent. Example of MAOP framework with 4 agents is shown in figure 4.

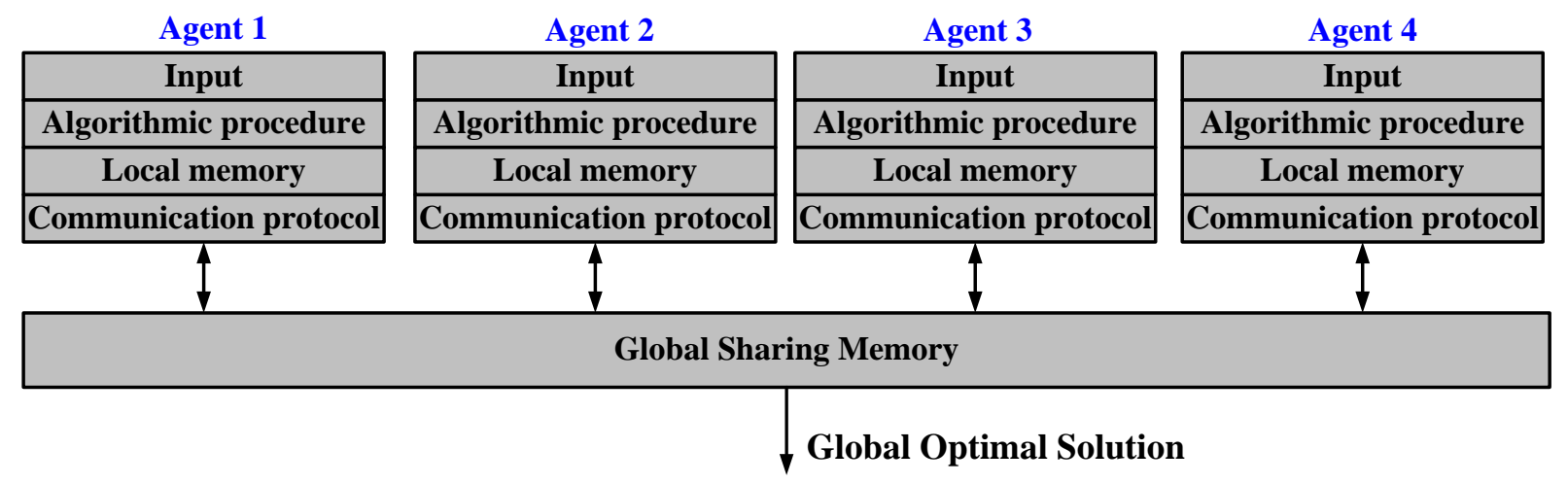

Figure 4: MAOP framework which has 4 agents.

\subsection{Parallelization}

Most algorithmic procedures are sequential, that is, sequences of steps are coded in which each step consists of a single operation. These algorithms are well suited to computers, which basically perform operations in a sequential fashion. Although the speed at which sequential computers operate has been improving at an exponential rate for many years, the improvement is coming at an increased cost. More cost-effective improvements are to use the parallel computers. These computers perform multiple operations in a single step. In order to solve a problem efficiently on a parallel computer, it is usually necessary to design an algorithm that specifies multiple operations on each step, i.e., a parallel algorithm Siirola et al. (2003). The parallelism in an algorithm improves the performance of parallel and single-processor computers. In the case of parallel computer, the operations in a parallel algorithm can be performed simultaneously by different processors Delisle et al. (2009). In a single-processor computer, the parallelism in an algorithm can be exploited by using multiple functional units, pipelined functional units, or pipelined memory systems. Thus, it is important to make a distinction between the parallelism in an algorithm and the ability of any particular computer to perform multiple operations in parallel.

In MAOP framework, beside of the message exchanging between the local and global sharing memories, there are two types of communication. The first deals with the resource allocation and the second handle the agent execution. These communications are controlled by the Matlab Job Scheduler (MJS) of the Matlab Distributed Computing Server (MDCS). Agents in MAOP framework may run on the same multiprocessor computer or on processors that reside 
on computers distributed over the network. As shown in figure 5, MJS is software that coordinates the execution of jobs (agent/algorithmic procedure) and resource allocation for their task. The MJS distributes the tasks for evaluation to the server's individual MATLAB sessions called processors (workers). The MJS runs jobs in the order in which they are submitted, unless any jobs in its queue are promoted, demoted, canceled, or destroyed. Each worker receives a task of the running job from the $M J S$, executes the task, returns the result to the $M J S$, and then receives another task. When all tasks for a running job have been assigned to workers, the $M J S$ starts running the next job with the next available worker (MathWorks, 2014).

The figure below illustrates the stages in the life cycle of a job. When a job is created and run, it progresses through a number of stages.

Pending: This is the state where the algorithmic procedure is ready to run and ready to be submitted to the scheduler for resource allocation and execution.

Queued: When algorithmic procedure submitted for the scheduler places the job in the queue, and the job's state is queued. The scheduler executes jobs in the queue in the sequence in which they are submitted, all jobs moving up the queue as the jobs before them are finished.

Running: When a job reaches the top of the queue, the scheduler distributes the job's tasks to worker sessions for evaluation. The job's state is now running. If more workers are available than are required for a job's tasks, the scheduler begins executing the next job. In this way, there can be more than one job running at a time.

Finished: When all of a job's tasks have been evaluated, the job is moved to the finished state. At this time, the results can be retrieved from all the tasks in the job.

Deleted: When a job's data has been removed from its data location, the state of the job in the client is deleted. 


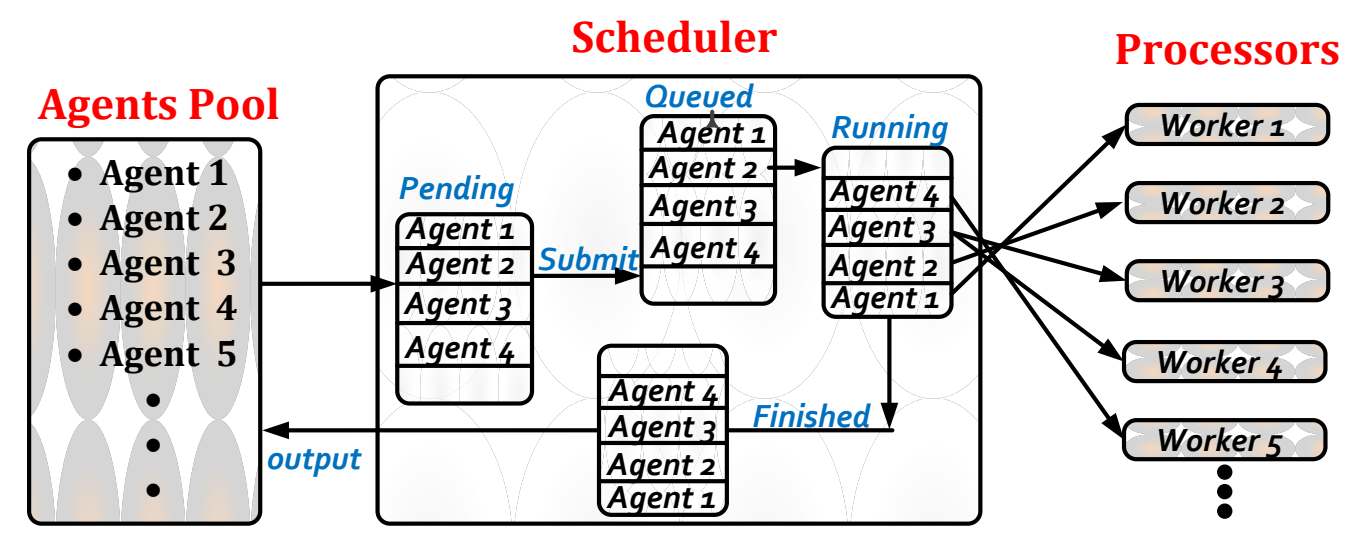

Figure 5: Matlab job scheduler

\section{Efficient ant colony optimization (EACO) algorithm}

Ant colony optimization (ACO) is a metaheuristic class of optimization algorithm inspired by the foraging behavior of real ants (Dorigo 1992). Natural ants randomly search food by exploring the area around their nest. If an ant locates a food source, while returning back to the nest, it lay down a chemical pheromone trail that marks its path. This pheromone trail will indirectly communicate with other members of the ant colony to follow the path. Over time, the pheromone will start to evaporate and therefore reduce the attraction of the path. The routes that are used frequently will have higher concentration of the pheromone trial and remain attractive. Thus, the shorter the route between the nest and food source imply short cycle time for the ants and these routes will have higher concentration of pheromone than the longer routes. Consequently, more ants are attracted by the shorter paths in the future. Finally, the shortest path will be discovered by the ant colony (Dorigo and Stutzle, 2004, and Zacchin et al., 2006). In ACO algorithm, artificial ants are stochastic candidate solution construction procedures that exploit a pheromone model and possibly available heuristic information of the mathematical model. The artificial pheromone trails (numeric values) are the sole means of communication among the artificial ants. Pheromone decay, a mechanism analogous to the evaporation of the pheromone trial of the real ant colony allows the artificial ants to forget the past history and focus on new promising search directions. Like the natural ants, by updating the pheromone values according to the information learned in the preceding iteration, the algorithmic procedure leads to very good and hopefully, a global optimal solution. 
A review on the general outline of ant colony algorithms to solve discrete optimization problems and the different variants of ant colony optimization can be viewed at Blum C. (2005). In this review paper, it has be also demonstrated how ant colony optimization can be applied to continuous optimization problems. Gebreslassie and Diwekar (2016) proposed EACO algorithm that improves the performance of the conventional ACO algorithm for combinatorial, continuous and mixed variable optimization problems by introducing the Hammersley sequence sampling technique (HSS) into the conventional ACO algorithm. The initial solution archive diversity for continuous and mixed variable optimization problems plays an important role in the performance of ACO algorithm. The uniformity property of the HSS technique is exploited to avoid clustering of the initial solution archive in a small region of the potential solution space (Diwekar and Ulas, 2007). Moreover, ACO algorithm is a probabilistic method; hence several random probability functions are involved in the algorithm procedure. For instance, the transition probability that help to choose the next solution component and the probability of choosing ant guide from the solution archive to construct and sample the Gaussian kernel require random numbers. The distribution of the random numbers generated for the acceptance probability of a solution component affects the performance of the ACO algorithm. At this stage, the multidimensional uniformity property of HSS is introduced to improve the computational efficiency of the ACO algorithm. The detail presentation of the EACO algorithms can be viewed (Gebreslassie and Diwekar, 2016). The major steps in EACO algorithm are shown in Table 1.

Process system engineering optimization problems are subjected to equality and inequality constraints. The EACO algorithm handles these constraints by implementing an Oracle penalty method proposed by Schluter et al. (2009). In general, constrained optimization problems are solved by transforming the problem into unconstrained optimization problem that is converting the original objective function into a penalty function. In most cases, the penalty function is given as a weighted sum of the original objective function and the constraint violations (Schluter et al. 2009). Thus, the penalty function serves as an objective function. The main advantage of the penalty method is its simplicity. The oracle penalty method is a generalized method capable of handling simple and challenging constrained optimization problems. Moreover, it is a simple method to implement. In the oracle penalty method, the objective function is first transformed into an additional equality constraint. The objective function becomes redundant in the transformed problem definition. Then minimizing the new constraint and minimizing the residual 
of the original equality and inequality constraints become directly comparable. Using a penalty function, the comparability property can be exploited. The penalty function balances its penalty weight each side of the transformed objective function and the violation of the original constraints. The implementation of the oracle penalty function can be referred found in Schluter and Gerdts (2010).

\section{Table 1: EACO Algorithm for mixed integer nonlinear optimization problems}

\section{Start program}

- Set $K, n A n t s, N C, N O, \rho, q$, $\xi$ and termination criteria

- Initialize solution archive $T(K, N C+N O)$ using HSS

- Combine and evaluate the objective function of the $K$ solutions $T(K, N D I M)$

- Rank solutions based on the quality of the objective function $\left(\mathrm{T}=\operatorname{rank}\left(S_{1} \ldots S_{K}\right)\right)$

\section{While Termination criterion is not satisfied}

- Generate solutions equivalent to the number of ants

For all \# nAnts

- Incremental solution construction

For all \# NDIM

- Probabilistically construct continuous decision variables

○ Probabilistically construct discrete decision variables

\section{End for \# NDIM}

- Store and evaluate the objective function of the newly generated solutions

\section{End for \# nAnts}

- Combine, rank and select the best k solutions, $\mathrm{T}=\operatorname{Best}\left(\operatorname{rank}\left(\mathrm{S}_{1} \ldots \mathrm{S}_{\mathrm{K}} \ldots \mathrm{S}_{\mathrm{K}+\mathrm{nAnt}}\right), \mathrm{K}\right)$

- Update solution

\section{End while}

\section{End program}

$T$ is solution archive and $k$ is size of T. nAnts is the number of ants. NC, and NDIM are the number of continuous, ordinal, categorical and the total number of decision variables, respectively.

\section{Benchmark problems}

Testing the proposed framework by solving the optimization problems of known solutions is an important step before applying the algorithm to solve real world optimization problems. Therefore, the main goal of solving the benchmark problems is to validate the proposed HMAO framework and compare the performance among the different classes of the algorithmic frameworks. The performance measures used to evaluate the algorithms are the quality of the 
solution and the elapsed time to reach the optimal solution for the similar algorithmic parameter setting and termination criterion. The benchmark problems are explained below and given in Table 2.

- Four benchmarks are used for the continuous optimization problems. The parabolic function is a convex function taken from Kim and Diwekar example I (Kim and Diwekar, 2002a). This function is a multidimensional parabolic function that has one global optimum at 0 at value of the decision variables equal to 0 . The three test functions, which are the ellipsoid, cigar and Rosenbrock functions are from Socha, (2009). The ellipsoid and cigar functions are convex functions while the Rosenbrock function is a nonconvex function.

Table. 2. Benchmarks problems

\begin{tabular}{lll}
\hline Function & Formula & Range \\
\hline Parabolic (PR) & $f_{P R}(x)=\sum_{\mathrm{i}-1}^{\text {NDIM }} \mathrm{x}_{\mathrm{i}}^{2}$ & {$[-5,5]^{N D I M}$} \\
Ellipsoid (EL) & $f_{E L}(x)=\sum_{\mathrm{i}=1}^{\mathrm{NDIM}} 5^{\frac{\mathrm{i}-1}{\mathrm{n}-1} \mathrm{x}_{\mathrm{i}}^{2}}$ & {$[-5,5]^{N D I M}$} \\
Cigar (CG) & $f_{C G}(x)=\mathrm{x}_{1}^{2}+10^{4} \sum_{\mathrm{i}=2}^{\mathrm{NDIM}} \mathrm{x}_{\mathrm{i}}^{2}$ & {$[-5,5]^{\text {NDIM }}$} \\
Rosenbrock (RB) & $f_{R B}(x)=\sum_{i=1}^{N D I M}\left(1-x_{i}\right)^{2}+100\left(x_{2 i+1}-x_{i}^{2}\right)^{2}$ & {$[-5,5]^{N D I M}$} \\
\hline
\end{tabular}

- An MINLP problem from Kim and Diwekar, (2002a) that has one global minimum of -1 and it is given below.

$$
f(x, y)=\sum_{i=1}^{N C}\left(x_{i}-i / N C\right)^{2}+\sum_{i=1}^{N D} y_{i}^{2}+\prod_{i=1}^{N D} \cos \left(4 \pi y_{i}\right)
$$

\subsection{Results of the benchmark problems}

All the computational studies are performed on ASUS Desktop PC M11AD series with Intel ${ }^{\circledR}$ Core $^{\mathrm{TM}}$ i5-4440S, 2.88-GHz CPU, and 6-GB RAM. This computer is networked with HP Z820 workstation with Intel ${ }^{\circledR} \mathrm{Xeon}^{\circledR} \mathrm{CPU}$ E5-2690 v2, 3.00-GHz, and 64-GB RAM. The termination criteria parameters are of two kinds. The local represents parameters that are only accessed by the individual agents and the global parameters are accessed only by the sharing memory environment. The HMAO algorithm terminates if the maximum global iteration 
MaxIter_gb is reached and/or if the global tolerance $\epsilon_{g b}$ that is the relative difference between solutions found in two consecutive global iterations is lower than or equal to the parameter $\epsilon_{g b}$ for a set of consecutive number of global iterations $I C O N \_g b$. The termination criteria for the individual agents are similar to the global iteration but differ in the parameter setting. The algorithm parameters used for the algorithmic agents are the archive sizes $\mathrm{K}$, the number of ants nAnts, EACO algorithm parameter q and the tolerance for global and local $\epsilon=1 \mathrm{E}-5$, evaporation parameter $\rho$, MaxIter_g $b=2000$, MaxIter_lc $=100$ (for NDIM $=25,50$ ) and 200 (for NDIM = $75,100), \quad I C O N \_g b=3$, and ICON_lc $=10$. The archive size increases as the number of decision variables increase. 25, 50, 75 and 100 decision variables are considered for each benchmark problem and hence each solution archive corresponds to the individual number of decision variables. The rest of the parameters that depend on the agents used in the MAOP framework are given in table 3 .

Table 3. Parameters setting of agents used in the MAOP framework

\begin{tabular}{ccccc}
\hline Agents & $\boldsymbol{q}$ & rho & Solution archive $($ K $)$ & nAnts \\
\hline Agent 1 & 1E-1 & 0.7 & $100(\mathrm{NDIM}=25,50) \& 200(\mathrm{NDIM}=75,100)$ & $\mathrm{K}$ \\
Agent 2 & $1 \mathrm{E}-2$ & 0.75 & $100(\mathrm{NDIM}=25,50) \& 200(\mathrm{NDIM}=75,100)$ & $\mathrm{K}$ \\
Agent 3 & $1 \mathrm{E}-2$ & 0.5 & $200(\mathrm{NDIM}=25,50) \& 200(\mathrm{NDIM}=75,100)$ & $\mathrm{K}$ \\
Agent 4 & 1E-3 & 0.6 & $200(\mathrm{NDIM}=25,50) \& 200(\mathrm{NDIM}=75,100)$ & $\mathrm{K}$ \\
\hline
\end{tabular}

The following 3 figures and 2 tables are results of the benchmark problems that compare the computational efficiency of standalone algorithm (this is single instance of the standalone algorithm), serial multi-agent framework (SMAO) and the HMAO frameworks.

In standalone algorithm, a single algorithmic agent is used to solve the optimization problem. Upon completion of the problem evaluation, the agent sends the current solution to the global sharing memory as an information message. In this framework, the global sharing memory is equivalent to the local sharing memory. Local sharing memory is a memory only accessed by the agent where the memory resides.

In the serial multi-agent system, the multiple agents are solved in a sequential manner and at each iteration, the solution of each agent is transferred to the global sharing memory. For the next iteration, each agent is initialized by propagating the new best solution from the sharing memory. 
In HMAO framework, as explained in the HMAO framework section, the multiple agents are independent jobs and solved in parallel. The multiple agents are created as independent jobs with a single task in a single job. The agents solve the same problem in parallel and cooperate through local communication protocol and the global sharing memory. However, depending on the number of jobs and the existing number of computer processors in the cluster, all agents do not need to run simultaneously and a processor might run several agents in succession. The results of an agent who completed the task evaluation send its new solution to the global sharing memory without the need to wait until all agents complete their respective task evaluation. If the optimality criterion is not satisfied, the results from the rest of the agents are retrieved and the sharing memory is updated accordingly and each agent is initialized by propagating the new best so far solution from the sharing memory.

Bullnheimer et al.(1998) proposed two communication strategies between the local and global sharing memory. In the first case, colony of ants share information at each cycle. In this case, the time needed for the global communication and synchronization implies a considerable overhead. In the second strategy, the goal was to reduce the overhead cost by allowing the algorithm perform a given number of cycles without exchanging information. The authors reach to the conclusion that the second strategy is preferable over the first one because it leads to a considerable reduction of the communication overhead. In this work, we follow the second strategy by Bullnheimer et al. (1998). Therefore, to reduce the co-operation overhead cost, each agent is allowed to run up to 100 iterations before sharing its results. However, it can also share the final information with the global sharing memory if there is no improvement in the solution of the local memory for consecutive 10 iterations.

The computational efficiency of the frameworks are measured based on the total elapsed time spent to get the global optimal solution. The y-axis of the figures represents the elapsed time and the $\mathrm{x}$-axis is the number of decision variables. These comparisons show us not only the computational efficiency of the frameworks across the different class of the optimization problems but also the computational efficiency is reflected upon the problem size.

Figure 6 presents the results of the parabolic function. All the solution methods were able to find the global optimal solution for each dimension that we have considered in this work. As we can see from the figure, the HMAO framework outperforms the standalone and serial multiagent frameworks. In general, if the problem size increases, the computational intensity increases 
that leads to an increment of the total elapsed time. The results in the figure shows that for parabolic function which is simple convex nonlinear optimization problems can be handled by a standalone optimization algorithm effectively.

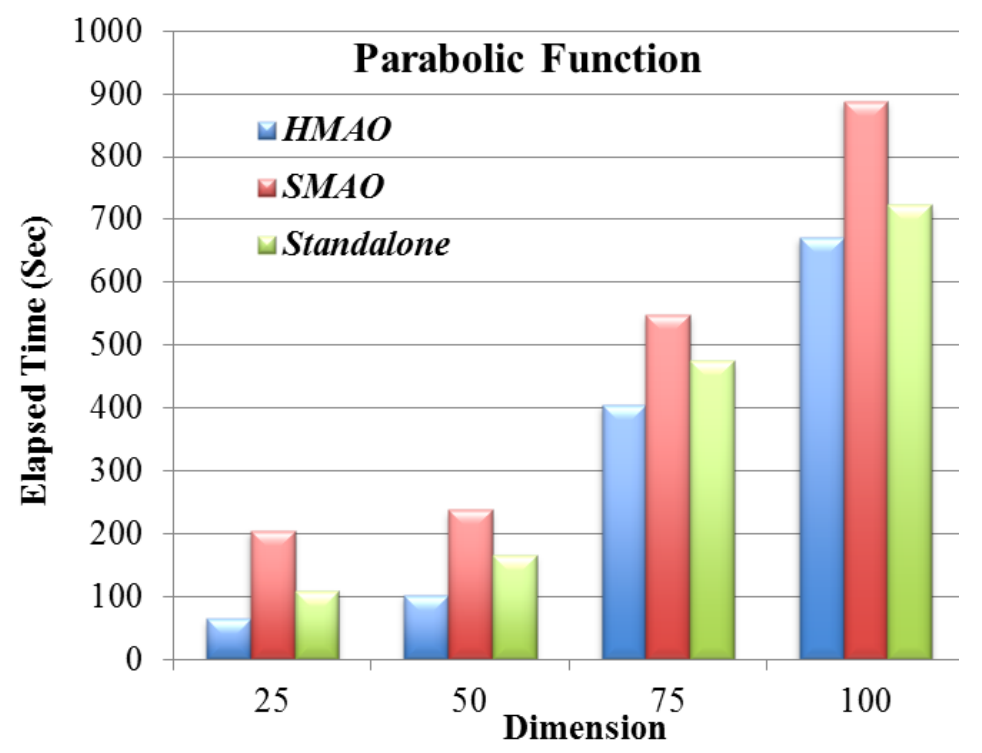

Figure 6: Computational efficiency of parabolic function as function of dimension

Figure 7 presents the results of the ellipsoid function which is a convex nonlinear function. As we can see from the figure, the HMAO framework shows a better computational efficiency compared to the SMAO framework and the standalone algorithm. However, similar to the parabolic function, the improvement is not significant. The performance of the SMAO framework is worst among all. For convex nonlinear problems, even though we can see improvements in the computational efficiencies, these types of problems are also can be handled by standalone algorithms. 


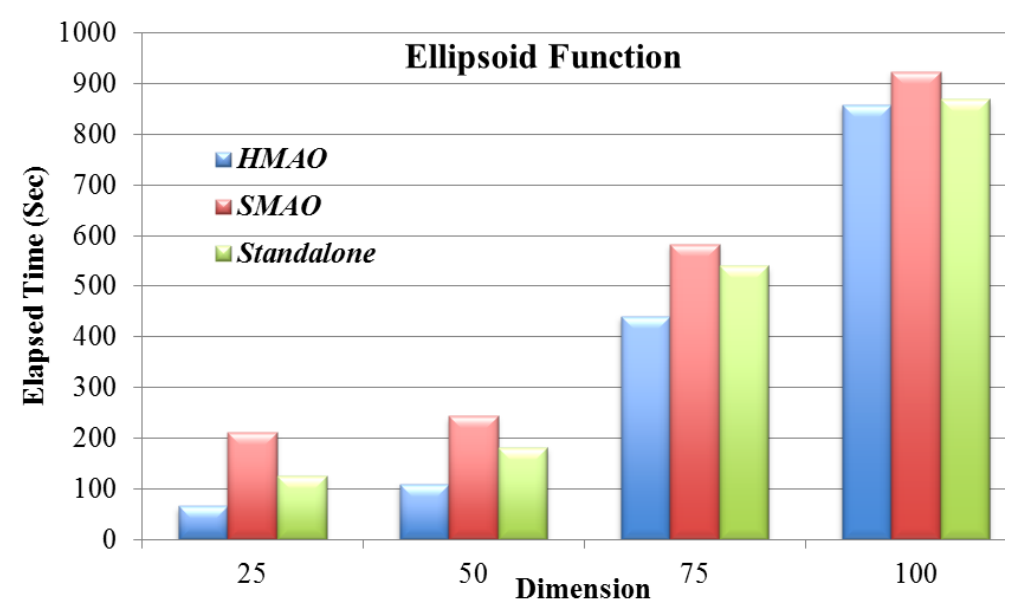

Figure 7: Computational efficiency of ellipsoid function as function of dimension

Tables 4 and 5 present results of the cigar and rosenbrock functions, respectively. Both functions have 0 global optimal solutions. Cigar function is a convex-quadratic function and rosenbrock function is a nonconvex optimization problem. The tables compare quality of the solution obtained from the HMAO, Serial multi-agent system (SMAO) and standalone algorithms. As we can see from table 4, up to 75 numbers of decision variables and within the termination criteria considered, all the three solution methods were able to find the global optimal solution. However, the standalone algorithm fails to find the global optimal solution as the number of decision variables increased to 100 . If we look at table 5 , for all number of decision variables that we considered here, the HMAO framework was either able find the global optimal solution or solutions close to the optimal solution. However, the serial multi-agent system (SMAO) fails to find the global optimal solution when the number of decision variables is 100. Moreover, the standalone algorithm was not able to find the global optimal solution for any number of decision variables. From these results, we can conclude that for nonconvex optimization problems, the standalone algorithm fails to find the global optimal solution and the serial multi-agent system was able to find optimal solutions for smaller problem size and close to global optimal solutions for large scale problems. Therefore, for nonconvex and nonlinear optimization problems the HMAO framework is an effective solution method.

Table 4: Solution quality comparison of cigar function solved using the HMAO, SMAO and the standalone algorithms.

NDIM Glb opt. HMAO SMAO Standalone




\begin{tabular}{ccccc}
\hline 25 & 0 & $6.99 \mathrm{E}-09$ & $4.50 \mathrm{E}-10$ & $3.13 \mathrm{E}-08$ \\
50 & 0 & $1.26 \mathrm{E}-07$ & $3.04 \mathrm{E}-07$ & $3.87 \mathrm{E}-07$ \\
75 & 0 & $3.36 \mathrm{E}-06$ & $1.83 \mathrm{E}-06$ & $6.48 \mathrm{E}-06$ \\
100 & 0 & $1.64 \mathrm{E}-06$ & $6.24 \mathrm{E}-06$ & 0.708242 \\
\hline
\end{tabular}

Table 5: Solution quality comparison of rosenbrock function solved using the HMAO, SMAO and the standalone algorithms.

\begin{tabular}{ccccr}
\hline NDIM & Glb opt. & HMAO & SMAO & Standalone \\
\hline 25 & 0 & $1.72 \mathrm{E}-05$ & $1.55 \mathrm{E}-05$ & 0.0005 \\
50 & 0 & $6.90 \mathrm{E}-06$ & $5.50 \mathrm{E}-05$ & 0.0001 \\
75 & 0 & $1.39 \mathrm{E}-05$ & $5.75 \mathrm{E}-05$ & 17.0260 \\
100 & 0 & $1.93 \mathrm{E}-05$ & $2.08 \mathrm{E}-04$ & 0.0082 \\
\hline
\end{tabular}

Figure 8 shows the results of the MINLP benchmark problem from Kim and Diwekar, (2002a). Across all ranges of the problem size, the HMAO framework outperforms the standalone algorithm and the serial multi-agent framework. In all cases, as the size of the problem increases the elapsed time increase because of the increase in the computational intensity. The HMAO framework beat the serial multi-agent and the standalone algorithms in terms of the computational efficiency. The improvement ranges from a factor of 1.483 for 50 decision variables to 1.655 of 100 decision variables. In almost all except one instance, the algorithms has found the global optimal (-1) solution. At the given parameter setting and 75 decision variables, the serial multi-agent framework was not able to find the global optimal solution (-0.876). Hence, the HMAO framework was able to beat its counter parts in terms of the computational efficiency performance and the quality of the solution. 


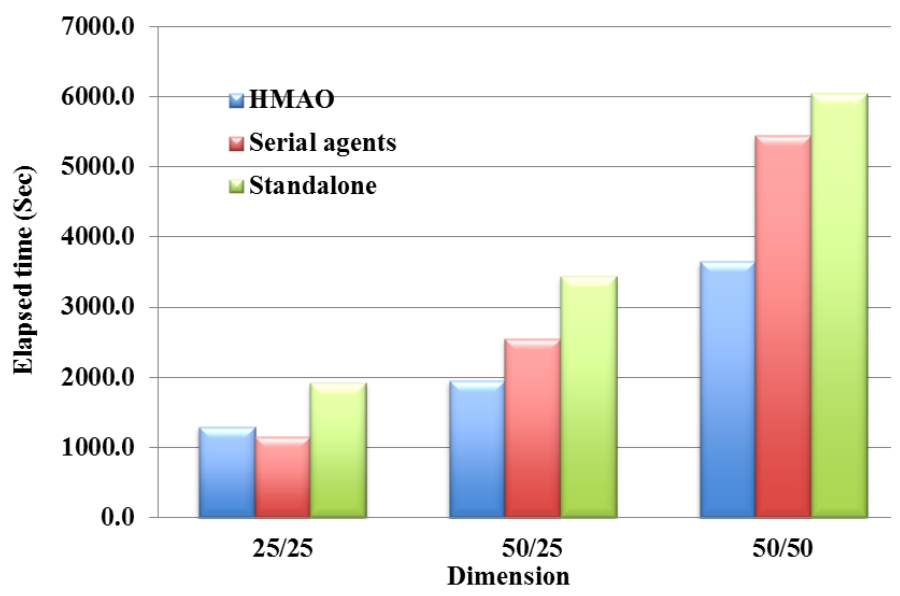

Figure 8: Computational efficiency an MINLP problem from Kim and Diwekar, (2002a). The nominator in the $\mathrm{x}$-axis represents the number of continuous and the denominator discrete decision variables.

From these results, we can conclude that the computational efficiency of the solution methods for large scale, nonconvex and mixed integer optimization can be effectively improved by multi-agent optimization framework. Moreover, significant improvement of the computational efficiency can be attained when agents are coordinated and solved in parallel. As shown in the results of the benchmark problems, the computational efficiency improvement is significant and it ranges from a factor of 1.01 in the case of the parabolic function to 1.9 of the ellipsoid function. We have also seen that as the problem size and complexity of the problem increases, the benefit of multi-agent framework and parallelization is more pronounced. However, the results also indicate that for the small size and convex nonlinear problems, there is no significant benefit in terms computational efficiency improvement from multi-agent system. This can be explained as; the overhead time spent for communication among agents through the sharing memory could be significant comparing to the computational time.

\section{Case study: Solvent selection problem}

\subsection{Computer added molecular design}

Computer aided molecular design (CAMD) for solvent selection is fast emerging systematic tool for efficient and reliable design of candidate solvents from their fundamental building blocks (Jorge Marrero, 2001, Karunanithi et al., 2005, Kim \& Diwekar, 2002bc, Xu and Diwekar, 2005). Beyond the solvent selection, the CAMD technique is practiced with great success in different disciplines such as pharmaceutical process designs (Gernaey and Gani, 
2009), polymer design (Satyanarayana et al., 2009), and bioethanol production (AlvaradoMorales et al. 2009). CAMD is generating large number of structural molecules with desired properties from a small set of structural groups (building blocks). CAMD is the reverse use the group contribution method. Different solution strategies are implemented to solve CAMD techniques: heuristic numeration (Hostrup et al. 1999, Li et al., 2002), knowledge based technique (Harper and Gani, 2000, Yamamoto and Tochigi, 2008), molecular property clusters with algebraic equations (Chemmangattuvalappil et al. 2009, Eljack and Eden, 2008, Kazantzi et al., 2007) and optimization-based methods (Diwekar and Shastri 2011, Karunanithi et al., 2005, Samudra and Sahinidis, 2005, Ostrovsky et al., 2002).

In the optimization approaches, because of the nonlinearity behavior of the UNIFAC model, the CAMD for solvent selection is formulated as a mixed integer nonlinear programming problem (MINLP) that seeks to optimize the desired properties of the solvent molecules subject to molecular design feasibility rules. To solve the MINLP formulation of the CAMD problems, different optimization methods have been proposed: decomposition methods that use local optimizers for the NLP sub-problem (Harper et al., 1999, Karunanithi et al., 2006, Karunanithi et al., 2005, Odele and Macchietto, 1993), global optimization (Ostrovsky et al., 2002, Samudra and Sahinidis, 2005), interval analysis (Achenie and Sinha, 2003), and dynamic optimization (Giovanoglou et al., 2003). Recently, probabilistic methods such as efficient ant colony algorithm (Gebreslassie and Diwekar, 2016), efficient simulated annealing (Kim and Diwekar, 2002a, b, c), and genetic algorithms (Cheng and Wang, 2008, Diwekar and Xu, 2005, Xu and Diwekar, 2005) and are adopted as an alternative to the gradient based decomposition methods.

Although the constraints related to the solvent selection have been already introduced in previous work by the authors Gebreslassie and Diwekar (2015), they are briefly introduced next for the sake of completeness of the overall mathematical formulation.

\subsection{Solvent Selection constraints}

The most important thermodynamic properties of solvents used for extraction process are solute distribution coefficient $m$, solvent selectivity $\beta$, solvent loss $S_{L}$, and physical properties like boiling point, density, viscosity.

Solute distribution coefficient $(m)$ : it is a measure of solvent capacity and it represents the solute distribution between the solvent and the raffinate phases. A high value of $m$ reduces the 
size of extraction equipment and the amount of recycling solvent. The solute distribution coefficient is estimated as by Eqn. 2 .

$$
m=\frac{\text { concentration of solute in extractive phase }}{\text { concentration of solute in raffinate phase }} \cong \frac{\gamma_{B A}^{\infty}}{\gamma_{B S}^{\infty}} \frac{M W_{A}}{M W_{S}}
$$

where $A, B$, and $S$ represent the raffinate (a nonpolluting molecule eg. water), solute (polluting molecule eg. acetic acid), and solvent (eg. ethanol) phases, respectively. MW denotes the molecular weight, and $\gamma^{\infty}$ is the infinite dilution activity coefficient. The definitions and constraints of the solvent selection problem are given below.

Solvent selectivity $(\beta)$ : It is defined as the ratio between the solute distribution coefficient and raffinate. It measures the potential of a solvent to selectively dissolve a solute (polluting molecule). A high solvent selectivity value leads to reduction in solute recovery cost and it is defined as shown in Eqn. 3.

$$
\beta=\frac{\text { distribution coefficient of solute }}{\text { distribution coefficient of solvent }}=\frac{m_{B}}{m_{A}} \cong \frac{\gamma_{A S}^{\infty}}{\gamma_{B S}^{\infty}} \frac{M W_{B}}{M W_{A}}
$$

Solvent loss $\left(S_{L}\right)$ : it is the concentration of solvent in raffinate phase i.e, the measure of the solvent loss tendency. A low solvent loss value means high selectivity toward solute and determines the immiscibility between the solvent and the raffinate. It is defined as shown in Eqn. 4.

$$
S_{L}=\text { concentration of solvent in raffinate phase } \cong \frac{1}{\gamma_{S A}^{\infty}} \frac{M W_{S}}{M W_{A}}
$$

In Eqns. 2, 3 and 4, the distribution coefficient, the solvent selectivity and the solvent loss properties are given as a function of the infinite dilution activity coefficients $\left(\gamma^{\infty}\right)$. It shows the non-ideality of the mixtures $(A-B, A-S$, and $B-S)$. If the mixture is ideal, $\gamma^{\infty}$ is close to one. Otherwise, tends to be greater than one or close to zero. $\gamma^{\infty}$ is a function of groups, temperature $T$, pressure $P$, and concentration. There are several group contribution methods for the prediction of $\gamma^{\infty}$. The most popular method is the UNIFAC group contribution method (Harper et al., 1999, Hostrup et al., 1999, Kim \& Diwekar, 2002c, Xu \& Diwekar, 2005). 
In the UNIFAC model, the activity coefficient $\left(\gamma_{i}\right)$ of a molecule $i$ have two parts: the combinatorial and residual part.

$\ln \gamma_{i}=\ln \gamma_{i}^{C}+\ln \gamma_{i}^{R}$

The combinatorial part reflects the volume and surface area of each molecule. The volume $q_{m}$ and surface area $r_{m}$ parameters of each group $m$ in molecule $i$ are involved in estimating the combinatorial part $\left(\gamma_{i}^{C}\right)$. The residual part represents the interaction energies of the molecules; hence, the volume parameter and interaction parameters $\left(a_{m n} ; a_{n m}\right)$ between groups $m$ and $n$ in the mixture are required to predict $\gamma_{i}^{R}$. The interaction parameters can be obtained by regression of experimental data of the mixture. The infinite dilution activity coefficient of molecule $i$ in mixture is limiting activity coefficient when the concentration of molecule $i$ in the mixture tends to zero. That is, $\gamma_{i}$ is function of the volume and surface area parameters, interaction parameters, temperature, pressure, and concentration. In this paper, the infinite dilution activity coefficients are calculated by the fifth revised original UNIFAC model (Hansen et al., 1991, Kim and Diwekar, 2002c) in which the original UNIFAC model is used with new groups and a revised data table. The UNIFAC model is summarized in the following Eqns. 6, 7, 8, and 9.

$$
\begin{aligned}
& \ln \gamma_{i}^{C}=\ln \left(\frac{\Phi_{i}}{x_{i}}\right)+\frac{z}{2} q_{i} \ln \left(\frac{\theta_{i}}{\Phi_{i}}\right)+l_{i}-\frac{\Phi_{i}}{x_{i}} \sum_{j} x_{j} l_{j} \\
& \ln \gamma_{i}^{R}=\sum_{k} v_{k}^{i}\left(\ln \Gamma_{k}-\ln \Gamma_{k}^{i}\right)
\end{aligned}
$$

$\ln \Gamma_{k}=Q_{k}\left[1-\ln \left(\sum_{m} \theta_{m} \psi_{m k}\right)-\sum_{m} \frac{\theta_{m} \psi_{k m}}{\sum_{n} \theta_{n} \psi_{n m}}\right]$

where

$\theta_{m}=\frac{Q_{m} X_{m}}{\sum_{m} Q_{m} X_{m}}, \quad \psi_{m n}=\exp \left(-\frac{a_{m n}}{T}\right), \quad \theta_{i}=\frac{q_{i} x_{i}}{\sum_{j} q_{j} x_{j}}, \quad \Phi_{i}=\frac{r_{i} x_{i}}{\sum_{j} r_{j} x_{j}}$

In these equations, $x_{i}$ is the mole fraction of component $i, \theta_{i}$ is the area fraction, $\Phi_{i}$ is the segment fraction, and $r_{i}$ and $q_{i}$ are measures of molecular van der Waals volume and molecular surface area, respectively. $\theta_{m}$ is the area fraction of group $\mathrm{m}, X_{m}$ the mole fraction of group $\mathrm{m}$ in 
the mixture, and $a_{m n}$ the group interaction parameter. The combinatorial part, $\ln \gamma_{i}^{C}$, is dependent on the sizes and shapes of the molecules, whereas the residual part, $\ln \gamma_{i}^{R}$, is dependent on group areas and group interactions.

Normal boiling point $\left(T_{b p}\right)$ : It is estimated using the following linear prediction model (Joback, 1987).

$T_{b p}=\sum_{i}^{N_{1}} t_{a}\left(N_{2}^{i}\right)+t_{b}$

The UNIFAC parameters in eqns. (6-9) and the boiling point group contribution parameters $t_{a}$ and $t_{b}$ shown in eqn. 10 are retrieved from Gebreslassie and Diwekar (2015).

For structural feasibility of a solvent configuration, the octet rule that relates the total number of free attachments of groups within the solvent molecule and the number of groups in the solvent molecule is used. Therefore, to determine the chemical feasibility the octet rule is used for acyclic groups as shown in Eqn. 11.

$\sum_{i}^{N_{1}} b_{i}=2\left(N_{1}-1\right)$

where $b_{i}$ is the number of free attachments in a group index $i$. Both high boiling point and low boiling point solvents are considered in the configuration.

\subsection{Solvent selection optimization model}

Acetic acid is commonly used as a process solvent and it is also a pollutant if released to the environment. Therefore, it is desirable to minimize the discharge of acetic acid to the environment. The most common method to recover or remove acetic acid from waste process streams is liquid-liquid extraction process. As shown in figure 11, the environmentally benign solvent (EBS) extracts acetic acid (HOAc) from the aqueous mixture that enters the extraction column. The extract is then supplied to the azeotropic distillation column where the bottom product is pure HOAc and the top product is a heterogeneous water-EBS azeotrope. The pure HOAc product is recycled to upstream processes, whereas the azeotropic mixture is condensed and then decanted. The organic phase from the decanter is recycled to the two columns, while the aqueous phase goes to the wastewater treatment facility. Because EBS can be lost at the effluent streams, fresh makeup EBS must be added continuously (Kim \& Diwekar, 2002d). 


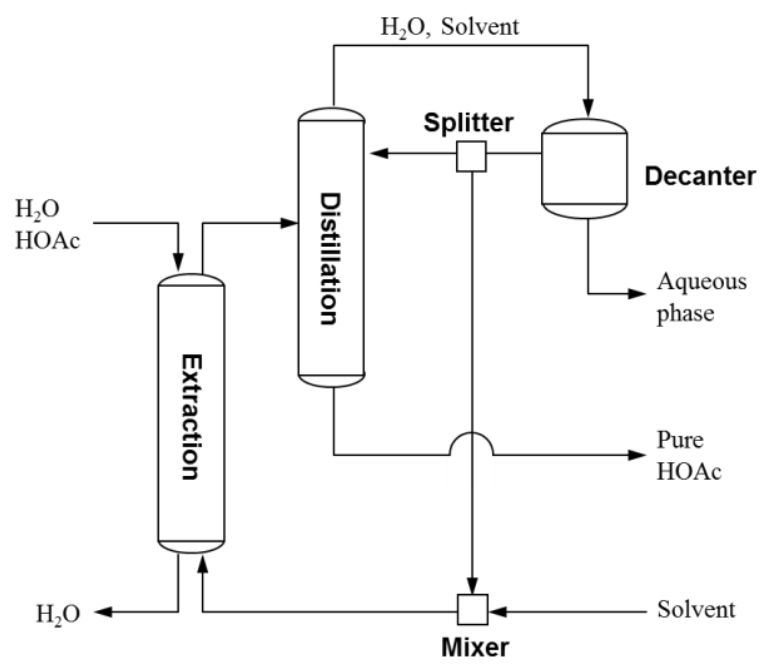

Figure 11: Acetic acid extraction process (Eastman Chemical Company) (Kim \& Diwekar, 2002d).

For the liquid-liquid extraction process, one can either use high boiling point solvents or low boiling point solvents (Joback, 1987; Kim \& Diwekar, 2002c). Ethyl acetate, isoamyl acetate, and isopropyl acetate are widely used in industries to extract acetic acid. Ethyl acetate, which is one of the common solvents for acetic acid extraction, has high solute distribution coefficient, $(\mathrm{m}=0.3156)$. Unfortunately, it also has high solvent loss $\left(\mathrm{S}_{\mathrm{L}}=0.0560\right)$. The high solvent loss property leads to high cost solvent recovering process. Therefore, the goal of this case study is to generate cost effective and environmentally benign candidate solvents with better solute distribution coefficient, selectivity and solvent loss. The molecular design problem is formulated as a mixed integer nonlinear programming (MINLP) model (Eqn. 12) in which a solute distribution coefficient is optimized subject to structural feasibility, property, and process constraints.

$$
\begin{array}{lll}
\min _{N_{1} N_{2}^{i}}- & \\
\text { s.t } \quad & \beta \geq \beta^{\min } & \\
& S_{L} \leq S_{L}^{\max } \\
& T_{b p}^{\min } \leq T_{b p} \leq T_{b p}^{\max } & \\
& 0 \leq N_{1} \leq 10 \\
& 0 \leq N_{2}^{i} \leq 24 \quad \forall i \in N_{1}
\end{array}
$$


The boundaries on the constraints are taken from the properties of the current practice of the solvent for acetic acid extraction (Xu and Diwekar, 2005) and can be viewed in Table 3. In this optimization problem, the discrete decision variables are the number of groups $N_{1}$ involved in a solvent molecule and the group index (the type of building blocks) $N_{2}^{i} ; i \in\left\{1, \ldots, N_{1}\right\}$ of that molecule. From the set of building blocks a unique solvent molecule that has maximum solute distribution coefficient and satisfies the selectivity, solvent loss, normal boiling point, thermodynamic and structural feasibility constraints can be generated.

The UNIFAC model is used to determine the objective function (solute distribution coefficient), and other solvent selection criteria such as solvent selectivity $(\beta)$ and solvent loss $\left(\mathrm{S}_{\mathrm{L}}\right)$, the normal boiling point. The total number of groups used in this work is 24 and a maximum of 10 groups per molecule are allowed, therefore, the total search space is $24^{10}(6.34 \times$ $10^{13}$ ) combinations. However, we have also tested the HMAO framework by increasing the maximum number of building blocks allowed to construct the molecule into 20 to test the computational efficiency of the algorithm as the problem size increases. The three UNIFAC parameters, surface area, volume, and interaction parameters, as well as boiling point parameters, group free attachments, molecular weights and the building groups' index are retrieved from the UNIFAC database. The detail of the UNIFAC model and the parameters needed for the CAMD formulation can be viewed in (Gebreslassie and Diwekar, 2015). 
Table 3 . Boundary constraints of the high and low boiling point solvents for acetic acid/water system.

\begin{tabular}{lc}
\hline \multicolumn{1}{c}{ Rank } & Property range \\
\hline \multicolumn{1}{c}{ High-Boiling-Point Solvent Constrains } & \\
Selectivity, $\beta_{\min }$ & 7 \\
Solvent loss, $S_{L}^{\max }$ & 0.01 \\
Boiling point temperature, $T_{\mathrm{bp}}(\mathrm{K})$ & $421-541$ \\
Number of groups in a molecule, $N_{1}$ & 0 - 10 \\
& 7 \\
Selectivity, $\beta_{\min }$ & 0.058 \\
Solvent loss, $S_{L}{ }^{\max }$ & $320.15-381.15$ \\
Boiling point temperature, $T_{\mathrm{bp}}(\mathrm{K})$ & $0-10$ \\
Number of groups in a molecule, $N_{1}$ & \\
\hline
\end{tabular}

\subsection{Results and Discussions}

The HMAO platform is written in MATLAB. All the computational studies are performed on an ASUS Desktop PC M11AD series with Intel ${ }^{\circledR}$ Core $^{\mathrm{TM}} i 5-4440 S$, 2.88-GHz CPU, and 6GB RAM. This computer is networked with HP Z820 workstation with Intel ${ }^{\circledR}$ Xeon ${ }^{\circledR}$ CPU E5$2690 \mathrm{v} 2,3.00-\mathrm{GHz}$, and 64-GB RAM. When two or more algorithmic frameworks are compared, we use same parametric setting and termination criteria. With the proposed framework, we are able to demonstrate the benefits from the multi-agent optimization and the parallelization of agents on the quality of the solutions and the computational efficiency. The results are divided into two major parts.

\subsubsection{Solvent selection results}

The total number of building blocks used in this work is 24 and maximum of 10 groups per molecule are allowed. Therefore, the search space is composed of $24^{10}\left(6.34 * 10^{13}\right)$ combinations. The HMAO algorithm terminates if the maximum global iteration MaxIter_g $b$ is reached and/or if the global tolerance $\epsilon_{g b}$ is lower than or equal to the parameter $\epsilon_{g b}$ for a set of consecutive number of global iterations $I C O N_{-} g b$. The termination criteria for the individual agents are similar to the global iteration but differ on the value of the parameter setting. The algorithm parameters used for the algorithmic agents are the archive sizes $K=50,100$, and 200, nAnts $=K$, 
$\mathrm{q}=1 \mathrm{E}-3$ and the tolerance for global and local $\epsilon=1 \mathrm{E}-5$, evaporation parameter $\rho=0.75$, MaxIter_g $b=2000$, MaxIter_lc $=200, I C O N \_g b=3$, and ICON_lc $=10$.

Depending on the extraction process objective; recycling or removal of acetic acid from waste process streams can use either use high-boiling point solvents or low boiling point solvents (Joback, 1987, Kim and Diwekar, 2002c). The constraints with respect to this high and low boiling point solvents are given in table 3 . The acetic acid extraction process used as case study is obtained from Eastman Chemical Company.

\subsubsection{Effect of solution archive size}

The authors have solved similar optimization problem using EACO algorithm (Gebreslassie and Diwekar, 2015). The size of the solution archive used to solve the problem was $\mathrm{K}=1500$. In this work, the HMAO framework was able to solve the problem with $\mathrm{K}$ value down to 50. And hence, in this work, we have also studied the effect of the size of the solution archive. We compare results of the benchmark problems using K values with 50, 100 and 200. For each $\mathrm{K}$ value, results from the HMAO, serial multi-agent system, and standalone algorithm are compared. Figure 11 shows that as the size of solution archive increase the elapsed time to solve the problem using all the optimization frameworks increase. Solution archive is the size of complete solutions stored at each iteration in which the probability distribution that the ants sample for the next iteration is constructed. Therefore, as size of the solution archive increases, the problem sizes increases and the computational burden increases. From the results, we have also absorbed that as the archive size increases the computational burden on the on the serial multi-agent framework is more pronounced. 


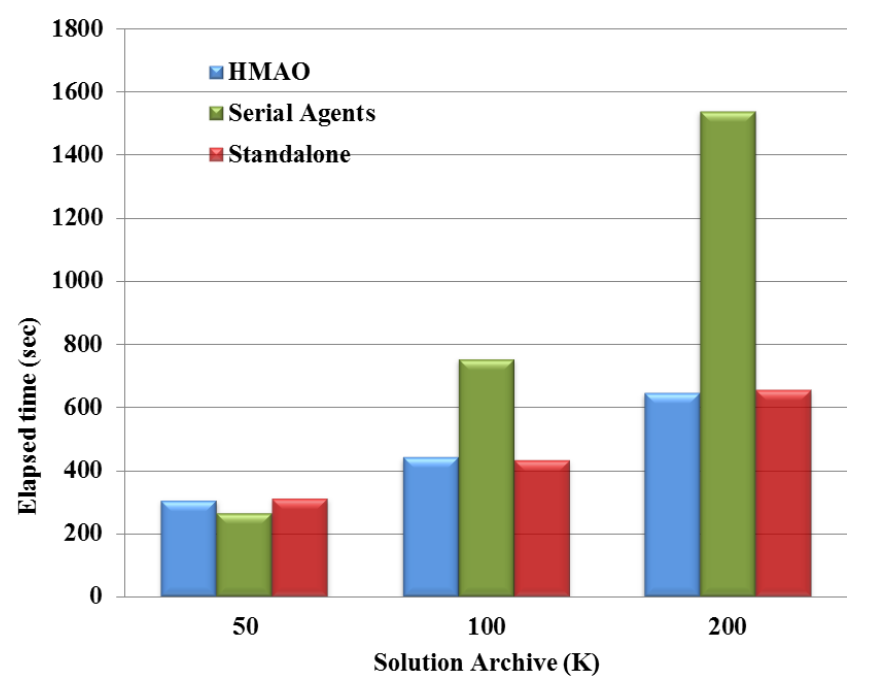

Figure 11: Computational efficiency of solvent selection problem as function of solution archive size.

\subsubsection{Effect problem size}

The optimization frameworks are also tested as function of the solvent selction problem size. The size of the combinatorial search space of the CAMD problem is increased by increasing the maximum number of possible groups to form the solvent molecule from 10 to 20. In this case, the combinatorial solution search space is increased from $6.34 \times 10^{13}$ to 4.02 $\times 10^{27}$ combinations. Figure 12 show results of the solvent selection problem for high boiling point solvents. As we can see from figure 12, all optimization frameworks show computational intensity increment indicated by increasing the computational and elapsed time to solve the solvent selection problem. However, the computational intensity shown by the standalone algorithm is by far higher than the rest of frameworks which is an increment by a factor of 10.3. That is the elapsed time to solve the CAMD problem using the standalone algorithm. It is increased approximately by 10.3 fold. The results of the HMAO and serial multi-agent frameworks have shown also a better quality of the global optimal solution of the solvent selection problem. The solute distribution coefficient of the optimal solvent with configuration of $\left(2 \mathrm{CH}_{3}, \mathrm{CH}_{2}, \mathrm{CH}, \mathrm{HCOO}\right)$ is 0.866 . This solution is obtained in both cases using the HMAO and the serial multi-agent frameworks. However, the standalone algorithm finds the global optimal solution at the reduced solution space that is when the maximum number of groups to construct the solvent molecule is restricted to 10 . However, when the maximum number of groups allowed to construct the solvent is increased to 20 , the standalone algorithm stuck in local optimal 
solution and finds a sub optimal candidate solvent with a configuration of $\left(3 \mathrm{CH}_{3}, \mathrm{C}, \mathrm{HCOO}\right)$ and solute distribution coefficient of 0.861 . From this we can conclude that as the optimization problem size increases the benefit of the HMAO framework is more pronounced not only in terms of the computational efficiency benefits but also in terms of the quality of the global optimal solution.

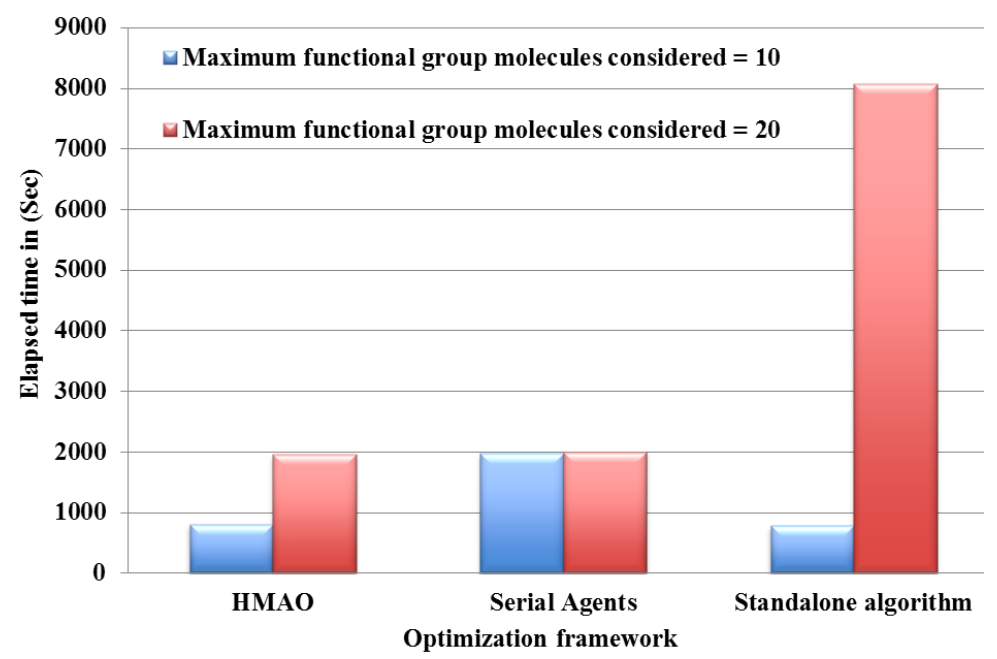

Figure 12: Computational efficiency of CAMD problem as function of problem size.

\subsubsection{Positioning HMAO framework}

In this section, we compare results of the high and low boiling point solvent selection problems obtained from HMAO framework. Tthe recent industrial practice, and results of similar problem that we get from the literature. The comparison is performed in terms of the quality of the optimal solutions. From the open literature, we find solutions of the solvent selection problems solved using a decomposition method (Karunanithi et. al. 2005) and metaheuristics such as efficient ant colony optimization (EACO) algorithm (Gebreslassie and Diwekar, 2015), efficient genetic algorithm (EGA) (Xu and Diwekar, 2005) and efficient simulated annealing (ESA) (Kim and Diwekar, 2002). Table 7 and table 8 show results of the high and low boing point solvents and the tables include the solution method, the configuration of the proposed solvent, the solute distribution coefficient $(\mathrm{m})$, the solvent selectivity $(\beta)$ and references. For the decomposition method and the current practice solvents didn't include the high and low boiling points of solvents and hence they are included in both tables for comparison. 
Before discussing the results, the authors would like to acknowledge that in the preceding work by the authors (Gebreslassie and Diwekar, 2005), there was a data discrepancy related to HCOO- building block so the group was not included in the calculation. In this work, the data discrepancy is corrected and the results discussed in this work also include candidate solvents that have the HCOO-functional group.

As shown in table 7 , the candidate solvent with configuration of $\left(2 \mathrm{CH}_{3}, \mathrm{CH}_{2}, 2 \mathrm{CH}\right.$, $\mathrm{CH}_{2}=\mathrm{C}, \mathrm{CH}_{3} \mathrm{CO}, \mathrm{HCOO}$ ) and solute distribution coefficient 1.296 and solvent selectivity of 27.6 is proposed from the HMAO framework. As shown in the table and to the best knowledge of the authors, this solvent is the best performance high boiling point solvent proposed so far in the literature. The closest best performance in terms of the thermodynamic properties candidate solvent is proposed from the EGA algorithm and EACO algorithm (after correction of the data discrepancy related to HCOO- building block) which is represented with $\left(4 \mathrm{CH}_{2}, \mathrm{CH}, \mathrm{CH}_{2}=\mathrm{CH}\right.$, $\mathrm{CH}_{3} \mathrm{CO}, \mathrm{HCOO}$ ) configuration and has solute distribution coefficient of 1.236 and solvent selectivity of 26.8. As shown in table 8, for the low boiling point solvents, the HMAO framework and the metaheuristic EACO, EGA and ESA algorithms find the global optimal candidate solvent with configuration of $\left(2 \mathrm{CH}_{3}, \mathrm{CH}_{2}, \mathrm{CH}, \mathrm{HCOO}\right)$, solute distribution coefficient of 0.865 and solvent selectivity of 24.1. Therefore, from these comparisons, we can conclude that the HMAO framework could be one of the best alternatives of optimization algorithm for solving solvent selection problems followed by the efficient genetic algorithm and efficient ant colony algorithms.

An attractive solvent should have a high solute distribution coefficient, high solvent selectivity and low solvent loss. These properties are used to screen out the most desirable candidate solvents proposed in this work. Accordingly, 4 best performing high boiling point candidate solvents are given in table 9 and 5 best performing low boiling point candidate solvents are enlisted in table 10. 
Table 7: Comparison of HMAO framework, decomposition method, EACO, EGA, and ESA for high boing solvents.

\begin{tabular}{|c|c|c|c|c|}
\hline Method & Solvent & $m$ & $\beta$ & References \\
\hline Industrial Practice & $2 \mathrm{CH}_{3}, \mathrm{CH}_{2}, \mathrm{COO}$ & 0.316 & 14.6 & - \\
\hline Decomposition & $1 \mathrm{CH}_{3}, 3 \mathrm{CH}_{2}, 1 \mathrm{CH}_{3} \mathrm{CO}$ & 0.491 & 11 & $\begin{array}{c}\text { Karunanithi et. al. } \\
\text { (2005) }\end{array}$ \\
\hline $\begin{array}{l}\text { Efficient Ant } \\
\text { Colony Algorithm }\end{array}$ & $2 \mathrm{CH}_{2}, \mathrm{C}, \mathrm{CH}_{2}=\mathrm{CH}, \mathrm{CH}=\mathrm{CH}, \mathrm{CH}_{3} \mathrm{OH}, \mathrm{CH}_{3} \mathrm{O}$ & 0.691 & 8.8 & $\begin{array}{l}\text { Gebreslassie and } \\
\text { Diwekar, (2015) }\end{array}$ \\
\hline $\begin{array}{l}\text { Efficient Genetic } \\
\text { Algorithm }\end{array}$ & $4 \mathrm{CH}_{2}, \mathrm{CH}, \mathrm{CH}_{2}=\mathrm{CH}, \mathrm{CH}_{3} \mathrm{CO}, \mathrm{HCOO}$ & 1.236 & 26.8 & $\begin{array}{l}\text { Xu and Diwekar, } \\
(2005)\end{array}$ \\
\hline $\begin{array}{l}\text { Efficient Simulated } \\
\text { Annealing }\end{array}$ & $\mathrm{CH}_{3}, 6 \mathrm{CH}_{2}, \mathrm{OH}$ & 0.607 & 19.8 & $\begin{array}{c}\text { Kim and } \\
\text { Diwekar, (2002) }\end{array}$ \\
\hline $\begin{array}{l}\text { Standalone } \\
\text { Algorithm }\end{array}$ & $4 \mathrm{CH}_{2}, \mathrm{CH}, \mathrm{CH}_{2}=\mathrm{CH}, \mathrm{CH}_{3} \mathrm{CO}, \mathrm{HCOO}$ & 1.236 & 26.8 & $\begin{array}{c}\text { ( EACO with } \\
\text { additional group) }\end{array}$ \\
\hline $\begin{array}{l}\text { Homogeneous } \\
\text { Multi-agent }\end{array}$ & $2 \mathrm{CH}_{3}, \mathrm{CH}_{2}, 2 \mathrm{CH}, \mathrm{CH}_{2}=\mathrm{C}, \mathrm{CH}_{3} \mathrm{CO}, \mathrm{HCOO}$ & 1.292 & 27.6 & This work \\
\hline
\end{tabular}

Table 8: Comparison of HMAO framework, decomposition method, EACO, EGA, and ESA for low boing solvents.

\begin{tabular}{|c|c|c|c|c|}
\hline Method & Solvent & $m$ & $\beta$ & References \\
\hline Industrial Practice & $2 \mathrm{CH}_{3}, \mathrm{CH}_{2}, \mathrm{COO}$ & 0.316 & 14.6 & Industrial practice \\
\hline Decomposition & $1 \mathrm{CH}_{3}, 3 \mathrm{CH}_{2}, 1 \mathrm{CH}_{3} \mathrm{CO}$ & 0.491 & 11 & $\begin{array}{r}\text { Karunanithi et. al. } \\
\text { (2005) }\end{array}$ \\
\hline $\begin{array}{l}\text { Efficient Ant Colony } \\
\text { Algorithm }\end{array}$ & $\mathrm{CH}_{2}=\mathrm{CH}, \mathrm{CH}_{2}=\mathrm{C}, \mathrm{CH}_{3} \mathrm{O}, \mathrm{CH}_{2} \mathrm{O}$ & 0.751 & 7.8 & $\begin{array}{l}\text { Gebreslassie and } \\
\text { Diwekar, (2015) }\end{array}$ \\
\hline $\begin{array}{l}\text { Efficient Genetic } \\
\text { Algorithm }\end{array}$ & $2 \mathrm{CH}_{3}, \mathrm{CH}_{2}, \mathrm{CH}, \mathrm{HCOO}$ & 0.866 & 24.1 & $\begin{array}{r}\text { Xu and Diwekar, ( } \\
\text { 2005) }\end{array}$ \\
\hline $\begin{array}{l}\text { Efficient Simulated } \\
\text { Annealing }\end{array}$ & $2 \mathrm{CH}_{3}, \mathrm{CH}_{2}, \mathrm{CH}, \mathrm{HCOO}$ & 0.866 & 24.1 & $\begin{array}{r}\text { Kim and Diwekar, } \\
(2002)\end{array}$ \\
\hline $\begin{array}{l}\text { Standalone } \\
\text { Algorithm }\end{array}$ & $2 \mathrm{CH}_{3}, \mathrm{CH}_{2}, \mathrm{CH}, \mathrm{HCOO}$ & 0.866 & 24.1 & $\begin{array}{r}\text { ( EACO with } \\
\text { additional groups) }\end{array}$ \\
\hline $\begin{array}{l}\text { Homogeneous Multi- } \\
\text { agent Framework }\end{array}$ & $2 \mathrm{CH}_{3}, \mathrm{CH}_{2}, \mathrm{CH}, \mathrm{HCOO}$ & 0.866 & 24.1 & This work \\
\hline
\end{tabular}




\subsubsection{Candidate solvents}

If we see the thermodynamic properties of those candidate solvents enlisted in table 9 and 10, all of them have higher solute distribution coefficient, high solvent selectivity, and low solute loss coefficient compared to the current practice of solvent (Ethyl acetate) for acetic acid extraction (refer to table 7 and 8). Moreover, the thermodynamic properties of the best high boiling point candidate solvent proposed in the literature is out performed by the top 3 candidate solvents proposed from HMAO framework. As shown in the tables, most of the high ranked solvent molecules are esters, ethers, and aldehydes. Hostrup et al. (1999) has enlisted 7 candidate solvent molecules for the extraction of acetic acid from water. The solute distribution co-efficient of these candidate solvents ranges from 0.11 (Methyl decanoate) to 0.5 (1-nonanol), which is relatively low compared to the result shown in tables 9 and 10. None of the 7 candidate solvents would make the candidate solvents listed in table 9 and 10.

A high value of the solute distribution coefficient reduces size of the extraction unit and the amount of recycling solvent. In other words, these high m solvents will reduce the investment cost and the operational cost related to the energy consumption of the solvent recovery. From Tables 9 and 10, it can be concluded that the HMAO framework can generate a set of candidate solvents with best solute distribution coefficient, solvent selectivity and low solvent loss properties. These candidate solvents may need further investigation related to environmental and toxicological properties and cost.

Table 9: High boiling point candidate solvents

\begin{tabular}{clcccc}
\hline Rank & \multicolumn{1}{c}{ Solvent } & $\boldsymbol{m}$ & $\boldsymbol{\beta}$ & $\boldsymbol{S}_{\boldsymbol{L}}$ & $\boldsymbol{T}_{\boldsymbol{b} \boldsymbol{p}}$ \\
\hline 1 & $2 \mathrm{CH}_{3}, \mathrm{CH}_{2}, 2 \mathrm{CH}, \mathrm{CH}_{2}=\mathrm{C}, \mathrm{CH}_{3} \mathrm{CO}, \mathrm{HCOO}$ & 1.292 & 27.63 & 0.008 & 539.2 \\
2 & $3 \mathrm{CH}_{3}, \mathrm{CH}, \mathrm{C}, \mathrm{CH}_{2}=\mathrm{C}, \mathrm{CH}_{3} \mathrm{CO}, \mathrm{HCOO}$ & 1.288 & 27.12 & 0.009 & 536.4 \\
3 & $2 \mathrm{CH}_{3}, \mathrm{CH}_{2}, \mathrm{C}, \mathrm{CH}_{2}=\mathrm{C}, \mathrm{CH}_{3} \mathrm{CO}, \mathrm{HCOO}$ & 1.2877 & 27.10 & 0.009 & 536.8 \\
4 & $\mathrm{CH}_{3}, \mathrm{CH}_{2}, \mathrm{CH}=\mathrm{CH}, \mathrm{CH}_{2}=\mathrm{C}, \mathrm{CH}_{2} \mathrm{CO}, \mathrm{HCOO}$ & 1.0388 & 20.12 & 0.010 & 521.3 \\
\hline
\end{tabular}

Table 10: Low boiling point candidate solvents 


\begin{tabular}{cllrll}
\hline Rank & \multicolumn{1}{c}{ Solvent } & $\boldsymbol{m}$ & \multicolumn{1}{c}{$\boldsymbol{\beta}$} & \multicolumn{1}{c}{$\boldsymbol{S}_{\boldsymbol{L}}$} & $\boldsymbol{T}_{\boldsymbol{b} \boldsymbol{p}}$ \\
\hline 1 & $2 \mathrm{CH}_{3}, \mathrm{CH}_{2}, \mathrm{CH}, \mathrm{HCOO}$ & 0.866 & 24.11 & 0.051 & 374.78 \\
2 & $\mathrm{CH}_{3}, \mathrm{CH}_{3}, \mathrm{CH}=\mathrm{CH}, \mathrm{HCOO}$ & 0.763 & 16.65 & 0.047 & 379.38 \\
3 & $\mathrm{CH}_{3}, \mathrm{CH}_{2}, \mathrm{CH}_{2}=\mathrm{C}, \mathrm{HCOO}$ & 0.748 & 15.23 & 0.050 & 371.78 \\
4 & $\mathrm{CH}_{2}=\mathrm{CH}, \mathrm{CH}=\mathrm{CH}, \mathrm{CH}_{3} \mathrm{O}$ & 0.709 & 9.25 & 0.019 & 337.18 \\
5 & $\mathrm{CH}_{3}, \mathrm{CH}=\mathrm{CH}, \mathrm{CH}_{3} \mathrm{CO}$ & 0.610 & 16.10 & 0.048 & 371.95 \\
\hline
\end{tabular}

\section{Conclusions}

In this paper, we proposed a novel homogenous multi-agent optimization (HMAO) framework for optimizing large scale process system engineering problems. HMAO framework is a nature-inspired optimization platform, which supports cooperative search by group of algorithmic agents connected through an environment with certain predefined information sharing protocol. To develop the HMAO framework, multiple efficient ant colony optimization (EACO) algorithms are considered as distinct algorithmic agents.

The proposed platform is validated through small and large scale problems and also tested on convex and nonconvex nonlinear (NLP), mixed-integer nonlinear (MINLP) benchmark problems and a computer-aided molecular design (CAMD) problem. The molecular design problem is a solvent selection for the extraction of acetic acid from waste process stream using liquid-liquid extraction. The UNIFAC model based on the infinite dilution activity coefficient is used to estimate the mixture properties and the problem is formulated as an MINLP. The HMAO framework maximizes the solute distribution coefficient of the solvent selection model formulation subject to structural feasibility, thermodynamic property relations, and process constraints. The model simultaneously determines the optimal decisions that include the size and the functional groups of the candidate solvents and the thermodynamic properties of the candidate solvents. The results show that solution quality and the computational efficiencies are improved significantly. Results of the benchmark problems show that as the complexity and problem size increase the benefits of the HMAO framework are more significant. The computational efficient improvement is ranged from 1.475 fold to 4.137 fold. The effect of parallelizing the cooperative agents shows a significant computational efficiency of the solver. 
The test on the computational efficiency and quality of the global optimal solution using the real world case study also shows that as size of the optimization problem increases, the benefit of the HMAO becomes more more significant. Specifically, as the number of groups allowed to construct a solvent increased from 10 to 20 , the elapsed time to solve the problem using the standalone algorithm is higher than the HMAO framework by a factor of 4.137. In terms of quality of solutions, high and low boiling point candidate solvents with high solute distribution coefficients, high solvent selectivity and low solvent loss properties are proposed using the HMAO framework.

\section{Acknowledgements}

We gratefully acknowledge the funding from DOE National Energy Laboratory grant \# DEFE0012451.

\section{References}

Aydemir FB, Günay A, Öztoprak F, Birbil SI, Yolum P. Multiagent cooperation for solving global optimization problems: an extendible framework with example cooperation strategies. J Glob Optim. 2013; 57:499-519.

Blum C. Ant colony optimization: Introduction and recent trends. Physics of Life Reviews 2005; $2,353-373$.

Bullnheimer B, Kotsis G, Strauss C. Parallelization Strategies for the Ant System. High Performance Algorithms and Software in Nonlinear Optimization, Kluwer, 1998; 24, 87-100.

Chemmangattuvalappil NG, Eljack FT, Solvason, CC, Eden, MR. A novel algorithm for molecular synthesis using enhanced property operators. Computers and Chemical Engineering 2009; 33, 636-643.

Cheng HC, Wang FS. Optimal biocompatible solvent design for a two-stage extractive fermentation process with cell recycling. Computers \& Chemical Engineering 2008; 32, 1385-96.

Crainic TG, Toulouse M. "Parallel Strategies for Meta-Heuristics." In F. Glover and G. Kochenberger (eds.), Handbook of Metaheuristics. Boston: Kluwer, 2003; pp. 475-513. 
Delisle P, Krajecki M, Gravel M. Multi-Colony Parallel Ant Colony Optimization on SMP and Multi-Core Computers. IEEE, 2009; 978-1-4244-5612-3.

Diwekar U, Xu W. Improved genetic algorithms for deterministic optimization and optimization under uncertainty. Part I. algorithms development. Industrial and Engineering Chemistry Research 2005; 44, 7132-7.

Diwekar UM, Kalagnanam JR. Efficient sampling technique for optimization under uncertainty. AIChE Journal 1997; 43, 440-7.

Diwekar UM, Shastri Y. Design for environment: a state-of-the-art review. Clean Technology \& Environmental Policy 2011; 13, 227-40.

Diwekar UM, Ulas S. Sampling Techniques volume 26. Encyclopedia of Chemical Technology. 2007.

Dorigo M. Optimization, learning and natural algorithms. PhD Thesis, Dept. of Electronics, Politecnico di Milano, Italy; 1992.

Dorigo M., \& Stutzle, T. (2004). Ant colony optimization theory. A Brandford Book, The MIT Press, Cambridge, Massachusetts; 2004.

Eljack FT, Eden MR. Systematic visual approach to molecular design via property clusters and group contribution methods. Computers \& Chemical Engineering 2008; 32, 3002-10.

Gebreslassie BH, Diwekar UM. Efficient ant colony optimization for computer aided molecular design: Case study solvent selection problem. Computers \& Chemical Engineering 2015; 78, $1-9$.

Gebreslassie BH, Diwekar UM. Efficient ant colony optimization (EACO) algorithm for deterministic optimization. International Journal of Swarm and Evolutionary Computation, 2016; 5(1), 1000131.

Gernaey KV, Gani R. A model-based systems approach to pharmaceutical product process design and analysis. Chemical Engineering Science 2010; 65, 5757-5769.

Giovanoglou A, Barlatier J, Adjiman CS, Pistikopoulos EN, Cordiner JL. Optimal solvent design for batch separation based on economic performance. AIChE Journal 2003; 49, 3095-109.

Han C, Douglas J, Stephanopoulos G. Agent-based approach to a design support system for the synthesis of chemical processes, Computers and Chemical Engineering 1995;19, 63-69. 
Hansen HK, Rasmussen P, Fredenslund A, Schiller M, Gmehling J. Vapor-liquid equilibria by UNIFAC group contribution. Industrial \& Engineering Chemistry Research 1991; 30, 23525 .

Harper P, Gani R. Design of environmentally benign processes: Integration of solvent design and separation process synthesis. Computers \& Chemical Engineering 1999; 23, 1395-414.

Harper P., Gani, R., Kolar, P., \& Ishikawa, T. (1999). Computer-aided molecular design with combined molecular modeling and group contribution. Fluid Phase Equilibria 1999; 158$160,337-47$.

Harper PM, Gani R. A multi-step and multi-level approach for computer aided molecular design. Computers \& Chemical Engineering 2000; 24, 677-83.

Holland JH. Adaptation in natural and artificial systems: An introductory analysis with applications to biology, control, and artificial intelligence. University of Michigan press: Ann Arbor, MI; 1975.

Julka N, Srinivasan R, Karimi I. Agent-based supply chain management-1: framework. Computers and Chemical Engineering, 2002a; 26, 1755-1769.

Julka N, Srinivasan R, Karimi I. Agent-based supply chain management-2: a refinery application. Computers and Chemical Engineering, 2002b; 26, 1771-1781.

Joback KG, Reid RC. Estimation of pure-component properties from group-contributions. Chemical Engineering Communications 1987; 57, 233-43.

Karunanithi AT, Achenie LEK. Gani R. A new decomposition-based computer-aided molecular/mixture design methodology for the design of optimal solvents and solvent mixtures. Industrial \& Engineering Chemistry Research 2005; 4, 4785-97.

Kazantzi V, Qin X, El-Halwagi M, Eljack FT, Eden MR. Simultaneous Process and Molecular Design through Property Clustering. Ind. Eng. Chem. Res 2007; 46, 3400-3409.

Kim K, Diwekar UM. Efficient combinatorial optimization under uncertainty. 1. Algorithmic development. Industrial and Engineering Chemistry Research 2002a; 41, 1276-84.

Kim K, Diwekar UM. Efficient combinatorial optimization under uncertainty. 2. Application to stochastic solvent selection. Industrial and Engineering Chemistry Research 2002b; 41, 1285-96. 
Kim K, Diwekar UM. Hammersley stochastic annealing: Efficiency improvement for combinatorial optimization under uncertainty. IIE Transactions Institute of Industrial Engineers 2002c; 34, 761-77.

Kim K, Diwekar UM. Integrated Solvent Selection and Recycling for Continuous Processes. Industrial \& Engineering Chemistry Research 2002d; 41, 4479-4488

MATLAB Parallel Computing Toolbox. User's guide, R2014a. MathWorks. 2014.

Mele F, Guillen G, Espuna A, Puigjaner L. An agent-based approach for supply chain retrofitting under uncertainty. Computers and Chemical Engineering 2007; 31,722-735.

Odele O, Macchietto S. Computer aided molecular design: a novel method for optimal solvent selection. Fluid Phase Equilibria 1993; 82, 47-54.

Ostrovsky GM, Achenie LEK, Sinha M. On the solution of mixed-integer nonlinear programming models for computer aided molecular design. Computers and Chemistry 2002; $26,645-60$.

Samudra AP, Sahinidis NV, Optimization-Based Framework for Computer-Aided Molecular Design. AIChE Journal 2013; 59(10), 3686-3701.

Schluter, M., Gerdts, M. (2010). The Oracle penalty method. Journal of Global Optimization 2010; 47, 293-325.

Schluter M, Gerdts M, Ruckmann JJ. A numerical study of MIDACO on 100 MINLP benchmarks. Optimization 2012; 61, 873-900.

Siirola JD, Hauan S, Westerberg AW. Computing Pareto fronts using distributed agents. Computers and Chemical Engineering 2004; 29, 113-126.

Siirola JD, Hauan S, Westerberg AW. Toward agent-based process systems engineering: proposed framework and application to non-convex optimization. Computers and Chemical Engineering 2003; 27, 1801-181.

Socha K. Ant Colony Optimization for Continuous and Mixed-Variable Domains. PhD Thesis, IRIDIA, CODE, Universit'e Libre de Bruxelles, CP 194/6; 2009.

Talukdar S, Baerentzen L, Gove A, de Souza P. Asynchronous Teams: Cooperation schemes for autonomous agents. Carnegie Mellon University Pittsburgh, PA 15213, 1996. 
Tatara E, Birol I, Teymour F, Cinar A. Agent-based control of autocatalytic replicators in networks of reactor. Computers and Chemical Engineering 2005; 29, 807-815.

Tetiker M, Artel A, Teymour F, Cinar A. Control of grade transitions in distributed chemical reactor networks-An agent-based approach. Computers and Chemical Engineering 2008; 32, 1984-1994.

$\mathrm{Xu}$ W, Diwekar UM. Improved genetic algorithms for deterministic optimization and optimization under uncertainty. Part II. Solvent selection under uncertainty. Industrial and Engineering Chemistry Research 2005; 44, 7138-46.

Zecchin A, Simpson A, Maier H, Leonard M, Roberts A, Berrisford M. Application of two ant colony optimization algorithms to water distribution system optimization. Mathematical and Computer Modelling 2006; 44, 451-68. 